\title{
Las transformaciones tecnológicas y el empleo en América Latina: oportunidades y desafíos
}

\author{
Jürgen Weller
}

\section{Resumen}

El debate sobre el impacto en el empleo de las transformaciones tecnológicas en curso se caracteriza por un elevado grado de incertidumbre, que se revela en las amplias discrepancias de las estimaciones sobre la posible destrucción de empleo. Aquí se revisan los aportes al análisis sobre la reestructuración de los mercados laborales y las proyecciones respecto de la destrucción, generación y transformación de empleo, y se argumenta en favor de un enfoque "contextual condicionado". Se enfatiza que, en comparación con los países desarrollados, en América Latina el impacto de las nuevas tecnologías estaría mediado por la estructura productiva y laboral, el desarrollo de la infraestructura, así como por las capacidades correspondientes. Finalmente, se analizan los desafíos en dos áreas clave para aprovechar el potencial de las nuevas tecnologías para el desarrollo sostenible con igualdad, a saber, las habilidades y competencias y la regulación de las relaciones laborales.

\section{Palabras clave}

Empleo, mercado de trabajo, cambio tecnológico, automatización, desempleo, creación de empleos, pronósticos de empleo, relaciones laborales, mano de obra, estadísticas del empleo, América Latina

\section{Clasificación JEL}

J23, J24, 033

\section{Autor}

Jürgen Weller fue Jefe de la Unidad de Estudios de Empleo de la División de Desarrollo Económico de la Comisión Económica para América Latina y el Caribe (CEPAL) hasta el 31 de diciembre de 2019. Correo electrónico: jurgenweller@gmail.com. 


\section{Introducción}

Las transformaciones tecnológicas en curso y proyectadas para el futuro próximo afectan a una amplia gama de actividades productivas y generan enormes oportunidades de desarrollo y bienestar, pero a la vez amenazas y desafíos, sobre todo respecto del empleo. La simultaneidad de oportunidades y amenazas genera un elevado grado de incertidumbre que se refleja en estimaciones extremadamente divergentes sobre la posible destrucción de puestos de trabajo.

Queda fuera de toda duda que estas transformaciones tecnológicas tendrán un profundo impacto en la dinámica de eliminación y surgimiento de puestos de trabajo y en las características de los empleos y las competencias requeridas. Adicionalmente, las relaciones laborales también se verán significativamente afectadas, dado que las modificaciones de los procesos productivos empujan hacia nuevas formas de organización del trabajo, tanto en el empleo asalariado como en modalidades laborales emergentes.

La magnitud de los retos se observa, por ejemplo, en la estimación - a menudo citada- de que un $65 \%$ de los niños que en la actualidad están iniciando sus estudios se desempeñarán en tipos de empleo y funciones que hoy en día ni siquiera existen (McLeod y Fisch, citado en Foro Económico Mundial, 2016, pág. 3), lo que subraya los desafíos que enfrentan los sistemas educativos y de formación profesional y capacitación.

Si bien las transformaciones tecnológicas no son los únicos procesos que influyen en el mundo de trabajo del futuro, todos estos elementos tendrán un gran impacto en el bienestar de los trabajadores y las sociedades en su conjunto, así como en la distribución de beneficios y costos que estos procesos conllevan.

En este artículo se propone analizar la influencia de las transformaciones tecnológicas en curso o futuras en los mercados laborales desde un enfoque contextual condicionado, que sostiene que este impacto depende en gran parte de las decisiones de los actores relevantes - aunque dentro de un margen establecido por las características propias de las tecnologías-. Este enfoque se distingue del enfoque compensatorio, que asegura que el funcionamiento de los mercados resultaría en la generación de nuevos empleos que compensarían la destrucción inicial de puestos de trabajo, y del enfoque determinista, que plantea que el impacto laboral del cambio tecnológico se desprende exclusivamente de las características de las tecnologías mismas.

En la siguiente sección se presentan algunas consideraciones generales sobre el impacto de las transformaciones tecnológicas en el empleo, y en la tercera sección se revisa la literatura sobre este impacto reciente. En la cuarta sección se examinan las proyecciones de destrucción y generación de empleo tanto a nivel global como específicamente para América Latina. En la quinta sección se examinan los retos de dos áreas claves para las políticas públicas que aspiran a influir en el impacto laboral de las transformaciones tecnológicas, a saber, la regulación de nuevas modalidades de relaciones laborales y el desarrollo de habilidades y competencias. En la última sección se resumen las principales conclusiones.

\section{Consideraciones generales}

Debido al potencial efecto sustitutivo para el trabajo humano que tienen las transformaciones tecnológicas, estas suelen generar, por un lado, preocupaciones respecto al surgimiento de un elevado desempleo de carácter tecnológico y, por el otro, expectativas respecto a mejoras en la calidad de vida relacionadas con fuertes reducciones del tiempo de trabajo (Mokyr, Vickers y Ziebarth, 2015). En cuanto al primer aspecto, Tarabusi (1997) diferencia tres tipos de desempleo según sus causas y duración: un desempleo de corta duración causado por cambios tecnológicos incrementales a nivel de la empresa, 
desempleo de mediana duración provocado por un cambio profundo a nivel sectorial y desempleo de larga duración como resultado de un cambio de paradigma tecnoeconómico que afecte la economía en su conjunto. Respecto al segundo aspecto, basta recordar el optimismo con el que Keynes, si bien advirtió respecto a la amenaza del desempleo tecnológico, celebró las transformaciones tecnológicas de su época por su impacto a largo plazo en la calidad de vida de futuras generaciones ${ }^{1}$.

Las transformaciones tecnológicas fueron fundamentales para el desarrollo capitalista de los siglos XIX y XX, principalmente por los marcados aumentos de productividad que generaron (por ejemplo, a través de la generalización del uso de las energías eléctrica, de vapor y de combustión, las diferentes máquinas introducidas en la industria textil y la línea de montaje). En el contexto de mercados cada vez más integrados, estos incrementos de la productividad y, en consecuencia, de la competitividad premiaron la introducción de las nuevas tecnologías y —con la excepción de algunos nichos específicoshicieron imposible sostener de manera competitiva la producción con las tecnologías previamente predominantes. En consecuencia, estos avances tecnológicos provocaron la eliminación de puestos de trabajo en ocupaciones vinculadas con procesos productivos que fueron quedando obsoletos.

Sin embargo, estas tecnologías no solo sustituyeron a otras en los procesos de elaboración de los mismos productos, sino que también facilitaron la aparición de una vasta gama de nuevos productos, tanto en el ámbito de la transformación de los procesos productivos y distributivos como en el del hogar (Gordon, 2016). En este contexto emergieron muchos nuevos empleos, que compensaron las pérdidas causadas por los procesos sustitutivos de mano de obra. Por lo tanto, la preocupación por el elevado desempleo que generaría el cambio tecnológico no se verificó en términos agregados. Además, gracias a las luchas sociales y políticas se produjeron algunos cambios laborales positivos, como la reducción de la jornada laboral, e incrementos salariales, facilitados por las ganancias de productividad².

Las transformaciones tecnológicas de la actualidad se caracterizan, entre otras cosas, por los avances en la computación cuántica, la Internet de las cosas, la interconectividad de la nube digital, la robótica, los macrodatos (big data), los vehículos autónomos y la inteligencia artificial (CEPAL, 2017). Este trabajo se centra, específicamente, en las transformaciones generadas por las tecnologías de la información y las comunicaciones (TIC), así como las ciencias cognitivas y tecnologías relacionadas que - hasta ahora y en el futuro cercano - han tenido el mayor impacto y pueden resumirse como la "universalización de la economía digital" (CEPAL, 2016, págs. 60-62).

Los avances en curso no se limitan a sectores específicos, sino que tienen un impacto en todas las ramas de actividad, y además contribuyen a una creciente integración entre ellas, difuminando sus límites. La delimitación entre los sectores secundario y terciario se vuelve cada vez más difusa; por ejemplo, las empresas productoras de bienes se están viendo obligadas a incorporar servicios a su oferta y en las tecnologías de la información y las comunicaciones la producción de hardware y software cada vez se integra más.

El impacto de las nuevas tecnologías en los procesos productivos se genera mediante "la creación de bienes y servicios digitales, la agregación de valor al incorporar lo digital en bienes y servicios en principio no digitales, y el desarrollo de plataformas de producción, intercambio y consumo" (CEPAL, 2016, pág. 61). Schwab (2016) sostiene que hay por lo menos tres razones por las cuales

\footnotetext{
Según Keynes, la gran tarea del futuro consistiría en usar el tiempo libre de manera sabia para vivir bien. Dijo que, durante mucho tiempo, el "viejo Adán" seguiría tan presente en cada uno de nosotros que todos tendríamos que seguir trabajando en alguna medida para mantenerlo contento. Sugirió que bastaría con turnos de tres horas, o una semana laboral de 15 horas, para acallarlo por un tiempo, ya que tres horas al día eran suficientes para satisfacer al "viejo Adán" en la mayoría de las personas. (Keynes, 1930, citado en Mokyr, Vickers y Ziebarth, 2015, pág. 41).

2 En varios países europeos estas transformaciones tecnológicas coincidieron con un marcado crecimiento demográfico (resultado de elevadas tasas de natalidad combinadas con un marcado descenso de la mortalidad, sobre todo la infantil), lo que incentivó flujos de emigración masivos, sobre todo hacia las Américas, en el siglo XIX y la primera mitad del siglo XX. De no existir esta "válvula de escape", la situación sociolaboral de muchos países europeos habría evolucionado de manera mucho más compleja, lo que sin duda habría afectado negativamente el desarrollo económico, social y político de estos países.
} 
estos cambios no serían una simple prolongación de la tercera revolución industrial: la velocidad, el alcance y el impacto en los sistemas de producción, gestión y gobernabilidad. Por lo tanto, la amplitud y profundidad de estos cambios - en parte, todavía potenciales - permiten hablar de una cuarta revolución tecnológica.

¿Qué nos enseñan las experiencias del pasado respecto al impacto de las transformaciones tecnológicas en los mercados laborales? ¿Con qué enfoque deberíamos estudiar el posible impacto de las nuevas tecnologías en el empleo, tanto respecto a su calidad como a su cantidad? Tarabusi (1997) ha identificado tres perspectivas de análisis:

- La perspectiva compensatoria plantea que, en mercados no distorsionados, las ganancias de eficiencia generadas por el cambio técnico se transforman en una mayor demanda de bienes de consumo (por su abaratamiento) y de capital (para introducir el mismo cambio técnico), lo que, además de reducir los costos salariales unitarios, incide en una mayor demanda laboral. De esta manera, el funcionamiento de los mercados compensa de forma automática las pérdidas de empleo causadas por el cambio técnico.

- Según la perspectiva determinista, las tecnologías tienen características intrínsecas que determinan su impacto, sin que realmente haya espacio para intervenir en la destrucción de empleos que generaría ni en las características que impondría a los puestos de trabajo.

- Finalmente, la perspectiva contextual enfatiza que los efectos de las nuevas tecnologías en la cantidad y en la calidad del empleo dependen del marco institucional y organizacional. El impacto del cambio técnico es variado y depende de procesos sociales y políticos en diferentes niveles, así como del contexto macroeconómico.

En este artículo se sostiene que las nuevas tecnologías generalmente no imponen una manera única de utilización, y su implementación no tiene un impacto lineal; la forma específica en que se usan se determina a través de mecanismos de regulación que resultan de procesos específicos en cada sociedad. Por otra parte, no se niega que, para que su aplicación sea eficiente en economías caracterizadas por mercados competitivos, el carácter de la tecnología suele imponer ciertos lineamientos y restricciones. En este sentido, se argumenta que un análisis adecuado parte de un enfoque contextual condicionado (un enfoque contextual absoluto supondría que a través del marco institucional y organizacional se lograría un manejo libre de las tecnologías). A la vez, se rechaza el determinismo tecnológico que niega que exista espacio para una regulación -legal o negociada entre empresas y trabajadores - de la aplicación de las tecnologías, así como la perspectiva compensatoria, ya que no se trata de que surja cualquier empleo, sino trabajo productivo y decente, tal como lo plantea el Objetivo de Desarrollo Sostenible 8.

\section{Las tendencias recientes: destrucción, creación y transformación del empleo}

El debate sobre el impacto futuro de las nuevas tecnologías debe tener en cuenta las transformaciones que han generado recientemente en los mercados laborales. Durante las últimas décadas, en los países desarrollados la destrucción de empleo que se relacionó, entre otros motivos, con el cambio tecnológico, se centraba en actividades rutinarias (cognitivas o manuales). Esto provocó una polarización de la estructura ocupacional, dado que se creó empleo en ocupaciones intensivas en tareas cognitivas no rutinarias y, por lo tanto, elevados niveles de educación, y en ocupaciones con tareas no rutinarias manuales, generalmente ejercidas por personas con niveles de educación formal más bajos (véase el gráfico 1$)^{3}$.

3 Véase la discusión de la evolución polarizada de la estructura ocupacional en los Estados Unidos en Acemoglu y Autor (2012). 


\section{Gráfico 1}

Países desarrollados: variación anual media de la proporción de los tipos de empleo según cualificación, alrededor de 1995 a alrededor de 2012

(En puntos porcentuales)

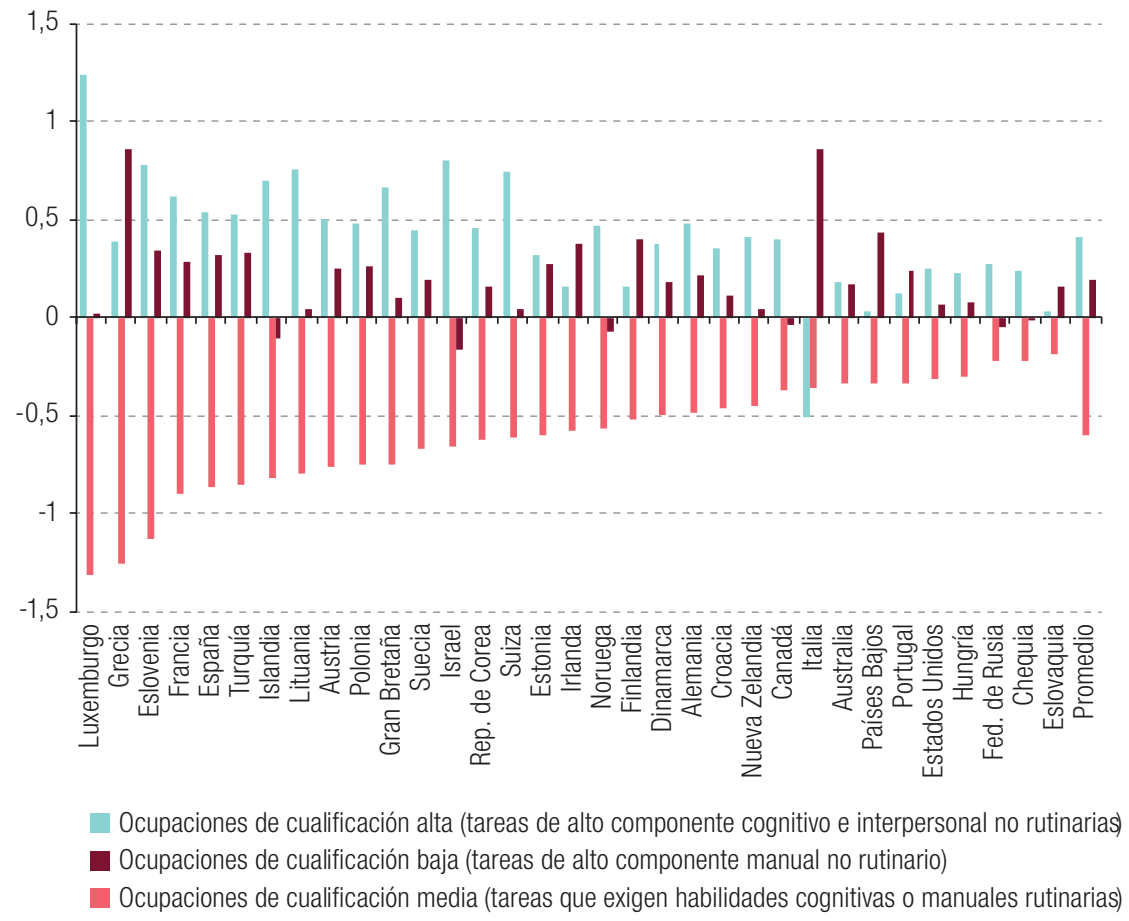

Fuente: Elaboración propia, sobre la base de datos de Banco Mundial, World Development Report 2016: Digital Dividends, Washington, D.C., 2016.

En el promedio de los países desarrollados, entre alrededor de 1995 y alrededor de 2012 las ocupaciones basadas en tareas rutinarias (tanto cognitivas como manuales) perdieron anualmente 0,6 puntos porcentuales en la estructura ocupacional, mientras que la participación de aquellas basadas en tareas cognitivas e interpersonales no rutinarias y en tareas manuales no rutinarias aumentó 0,4 y 0,2 puntos porcentuales, respectivamente. Esta reestructuración ocupacional responde, entre otros factores, al cambio tecnológico, pues tanto en la industria manufacturera como en los servicios el mayor uso de las tecnologías de la información y las comunicaciones está positivamente correlacionado con la proporción de empleo no rutinario (OCDE, 2017, pág. 46).

También entre los países de ingresos bajos y medianos se encuentra esta polarización, si bien no de manera tan generalizada (Banco Mundial, 2016). En el gráfico 2 se muestran los resultados correspondientes a los países con información de América Latina y el Caribe.

En el promedio del conjunto de los países de la región con información disponible, el segmento intermedio perdió 0,4 puntos porcentuales en la estructura de ocupaciones, mientras que la participación de los segmentos alto y bajo aumentó 0,3 y 0,1 puntos porcentuales, respectivamente.

El resultado de un ejercicio similar ejecutado en el período 1995-2015 por la Organización Internacional del Trabajo (OIT) (2016c, pág. 55) en un conjunto de 30 países de América Latina y el Caribe fue ligeramente diferente. Si bien también se observa que la mayor tasa de crecimiento (más del 3,5\% por año) correspondió a la expansión de las ocupaciones centradas en tareas cognitivas no rutinarias, según este estudio las ocupaciones con un alto componente rutinario crecieron más que las manuales no rutinarias $(2,3 \% \text { y } 1,6 \% \text {, respectivamente) })^{4}$. En cualquier caso, se confirma cierta

\footnotetext{
4 Estos datos se basan en cálculos y estimaciones realizados por la OIT (2015) e incluyen algunos países de mayor tamaño que no están cubiertos por el estudio del Banco Mundial, como el Brasil, Colombia y Venezuela (República Bolivariana de).
} 
tendencia hacia empleos de mayores niveles de cualificación. El estudio de Aboal y Zunino (2017) sobre el impacto de la innovación en la industria manufacturera de tres países latinoamericanos confirma esta tendencia, pues en dos de estos países (Argentina y Uruguay) dicha innovación tiene un mayor impacto positivo en la generación de empleos cualificados que en la de los no cualificados, mientras que en Costa Rica el impacto en ambos niveles de cualificación es muy similar.

\section{Gráfico 2}

América Latina y el Caribe: variación anual media de la proporción de los tipos de empleo según cualificación, alrededor de 1995 a alrededor de 2012

(En puntos porcentuales)

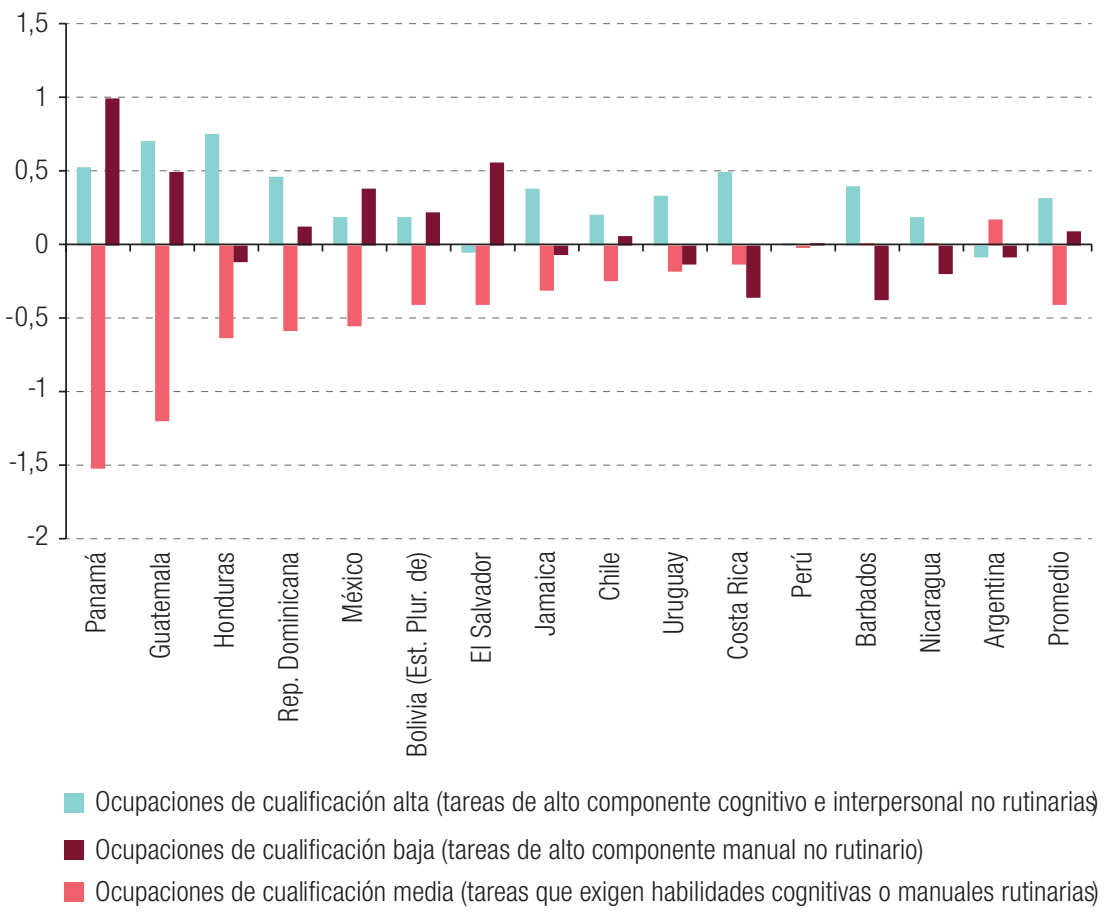

Fuente: Elaboración propia, sobre la base de datos de Banco Mundial, World Development Report 2016: Digital Dividends, Washington, D.C., 2016.

Al igual que en las revoluciones tecnológicas precedentes, paralelamente a la pérdida de empleos causada por las transformaciones surgen nuevos puestos de trabajo en un espacio que frecuentemente había sido subestimado. Acemoglu y Restrepo (2016) sostienen que son precisamente el cambio tecnológico y los procesos de automatización los que están generando nuevas ocupaciones de complejidad creciente. Según estos autores, entre 1980 y 2007 aproximadamente la mitad de los empleos surgidos en los Estados Unidos correspondían a ocupaciones que no existían en 1980. Esto indicaría que el potencial para generar nuevos empleos difícilmente es predecible. Por otro lado, los autores argumentan que existirían procesos de autocorrección: la automatización tiende a bajar los salarios, por lo que la generación de nuevos empleos se vuelve más rentable, aunque existen ineficiencias tanto en los mercados de productos como de trabajo que pueden bloquear este proceso. Además, si las transformaciones tecnológicas, por sus propias características, aceleran el desarrollo de nuevas innovaciones, esto podría resultar más desfavorable para el factor trabajo. En efecto, en un análisis empírico los mismos autores concluyen que los robots tienen un impacto negativo significativo en los mercados laborales locales de los Estados Unidos, tanto para el empleo como para los salarios, de manera que un posible efecto compensatorio no habría alcanzado para crear empleos de la misma cantidad y calidad que los puestos de trabajo destruidos (Acemoglu y Restrepo, 2017). 
Graetz y Michaels (2017) confirman los resultados previos según los cuales en los Estados Unidos el cambio tecnológico estuvo relacionado con la baja intensidad laboral de recientes reactivaciones poscrisis a partir de 1990, ya que esta baja intensidad laboral se dio, sobre todo, en sectores con elevadas proporciones de tareas rutinarias, más afectadas por la automatización. Sin embargo, no observan esta asociación en otros países desarrollados.

Además de los aspectos estructurales, también la situación de la coyuntura económica incide en el impacto inmediato de las transformaciones tecnológicas en el agregado. Según sea la situación de la coyuntura - a la que contribuye el dinamismo generado por las transformaciones tecnológicas - , la aparición de nuevos empleos compensará en mayor o menor grado la pérdida de ciertos puestos de trabajo. En los Estados Unidos, Aaronson y Phelan (2017) observaron que, entre las ocupaciones de bajos salarios, aquellas que eran predominantemente rutinarias con contenido de conocimiento tenían más posibilidades de ser reemplazadas. En el contexto de una expansión bastante dinámica de la economía en general, muchas de las personas que perdieron su empleo en este tipo de ocupaciones lograron volver a emplearse, aunque con pérdidas en la calidad (salarial) del empleo.

Otro ejemplo empírico se encuentra en Hathaway y Muro (2016), que analizaron el impacto de la introducción de plataformas de negocios en las áreas de transporte de personas y alquiler temporal de viviendas en los Estados Unidos. Los autores concluyeron que, a medida que se expandía el empleo vinculado a estas plataformas, los puestos de trabajo relacionados con las actividades tradicionales de este campo no solo no decayeron, sino que también crecieron, aunque a menor ritmo.

En ciertas circunstancias, las mismas transformaciones tecnológicas, pese a ser en sí ahorradoras de mano de obra, facilitaron la generación de nuevos empleos. Bessen (2015) cita el caso de la introducción de los cajeros automáticos, que supuestamente iba a conllevar una fuerte reducción del empleo en los servicios financieros, sobre todo en el caso de los cajeros. Sin embargo, la reestructuración y expansión del negocio (en parte debido a la reducción de costos causada por el cambio tecnológico) implicó el surgimiento de nuevas tareas y provocó que el número de empleados bancarios, de hecho, aumentara.

De esta manera, la evolución del empleo depende no solo del impacto de las transformaciones tecnológicas, sino también del contexto general de la economía. En los países desarrollados, esta últimamente se encuentra en una fase expansiva, pero no está garantizado que esta generación de empleo vaya a continuar. Sin embargo, se puede constatar que, en una perspectiva dinámica, el impacto de estas nuevas tecnologías en el empleo es más complejo que la simple sustitución de empleos por máquinas o por otros tipos de trabajo, como se suele afirmar desde una perspectiva de determinismo tecnológico.

\section{Perspectivas de cambio en el empleo y las ocupaciones}

Antes de examinar los resultados de algunos de los estudios dedicados al análisis del impacto de las transformaciones tecnológicas en el empleo, hay que recordar que la evolución del empleo, además de ser afectada por el contexto macroeconómico recién destacado, se ve influida por otras tendencias de largo plazo, como el cambio demográfico, la expansión de las economías y la reestructuración de los aparatos productivos (por ejemplo, por la evolución de las cadenas de valor y los esfuerzos de descarbonización)5.

\footnotetext{
5 En los Estados Unidos, la Oficina de Estadísticas Laborales proyectó que en el período 2016-2026 la mayor cantidad de nuevos empleos surgiría en los rubros de salud, operaciones de negocios y financieras, preparación y servicio de comida y cuidado personal (citado en Foro Económico Mundial, 2018). El McKinsey Global Institute (2017b) estima, para seis países de diferentes niveles de ingreso per cápita, el impacto neto de la automatización y, además, de la generación de nuevos empleos como consecuencia de varias de estas tendencias.
} 
Algunos expertos coinciden en que las ocupaciones en peligro seguirán siendo las que tienen un alto componente de trabajo rutinario. Por ejemplo, el McKinsey Global Institute (2017a) argumenta que las actividades que podrían verse más afectadas por la automatización son aquellas que requieren un nivel medio de cualificación. Entre ellas destacarían las actividades físicas predecibles y la operación de maquinaria en un entorno predecible, la recolección de datos y su procesamiento como actividades con el más alto potencial de automatización. En este sentido, las transformaciones tecnológicas mantendrían los procesos de polarización de la estructura ocupacional que han caracterizado sobre todo a los países desarrollados durante el período reciente. También KPMG International Cooperative (2016) proyecta que los empleos rutinarios de ingreso medio serían los que más se verían sustituidos en el futuro. Manpower Group (2017) sostiene que los empleos amenazados se concentrarán en las ocupaciones de cualificación baja e intermedia. Los principales perdedores de las nuevas transformaciones productivas y laborales serían, concretamente, las personas con un bajo nivel de cualificación, sobre todo si existen obstáculos para que adquieran los conocimientos y habilidades para los que estarían surgiendo nuevas opciones de empleo.

Otro grupo cuyo empleo puede verse afectado de forma considerable en este contexto son las mujeres. Una gran proporción de ellas se desempeñan en ocupaciones de cualificación media amenazadas por la sustitución tecnológica - por ejemplo, en trabajos administrativos, de ventas y de operaciones financieras -, mientras que, por otro lado, se encuentran subrepresentadas en las carreras universitarias y algunas de las ocupaciones con potencial de expansión ${ }^{6}$.

Por otra parte, otros analistas destacan en particular el impacto transversal que pueden tener las transformaciones tecnológicas presentes y futuras en una amplia gama de ocupaciones (Krull, 2016, pág. 20). Sostienen que, más allá de las actividades rutinarias que estuvieron en el centro de los procesos de automatización en el pasado, estas transformaciones también van a afectar, por un lado, a actividades de cualificación media o baja que tienen un elevado componente no rutinario (comercio, transporte o servicios sociales, entre otras) y, por otro, a tareas que requieren niveles intermedios o elevados de cualificación. Ejemplos de ello son los procesos de automatización de muchas tareas administrativas; la ampliación de capacidades en el área de sensores, que aumentaría significativamente la movilidad de máquinas, y el desarrollo de la inteligencia artificial y de sus capacidades de aprendizaje - por medio de la adquisición de información sin intervención humana- y de toma de decisiones con base en probabilidades, procesos facilitados por el desarrollo exponencial de la tecnología de hardware (Pratt, 2015).

La introducción de nuevas tecnologías ha tenido lugar en un mundo globalizado, que se caracteriza por una internacionalización de la producción representada por cadenas de valor globales que integran componentes del proceso productivo localizados en diferentes países. Las consideraciones sobre el posible impacto de las transformaciones tecnológicas deben tomar en cuenta los efectos que pueden tener estas transformaciones sobre las cadenas de valor.

Al respecto se pueden identificar dos tendencias opuestas. Por un lado, al abaratar el costo del capital, las nuevas tecnologías modifican los precios relativos con los que surgieron estas cadenas, al mismo tiempo que aumentan el peso de los componentes cognitivos. Además, la distancia entre los componentes de la cadena, y también entre su último componente y el mercado final, puede llegar a ser un obstáculo. En este contexto específico se reduciría el peso de la mano de obra barata como factor de producción. Desde hace algunos años ya se identifican indicios de reversión de algunos procesos de relocalización: se han traído de vuelta algunas unidades de producción desde países de salarios bajos hacia países desarrollados, sobre todo en el contexto del menor crecimiento económico global

\footnotetext{
6 Véase OCDE (2017). Según estimaciones de la Oficina de Estadísticas Laborales de los Estados Unidos, un 57\% de los empleos que se eliminarían en ese país entre 2016 y 2026 corresponden a mujeres (citado en Foro Económico Mundial, 2018). Esto contrasta con la pérdida de empleos registrada anteriormente, sobre todo en la industria manufacturera, que afectó principalmente a los hombres (Miller, 2017).
} 
(Kinkel, 2012; Saxer, 2017). La pérdida de dinamismo del comercio mundial podría estar reflejando este cambio en las estrategias de muchas empresas y una situación en que prácticamente todas las industrias intensivas en mano de obra que no se han automatizado ya se han trasladado desde países desarrollados a otros con costos laborales más bajos (Wolf, 2016; OIT, 2016a).

Por otro lado, los procesos de externalización (outsourcing) de actividades de servicios como el apoyo administrativo, las operaciones financieras, la atención a consumidores u otras se verían fortalecidos por plataformas de trabajo que faciliten la subcontratación externa de trabajos cualificados en una gran variedad de áreas de trabajo. Existen diferentes modalidades de externalización, tanto por medio de equipos definidos como a través de la externalización abierta de tareas (crowdworking) o modelos similares. De esta manera pueden surgir nuevas interacciones globales - por ejemplo, en el campo de resolución de problemas - en forma de redes, en lugar de cadenas.

En los últimos años se han realizado una serie de estimaciones cuantitativas sobre la destrucción y la generación de empleo a partir de la introducción de nuevas tecnologías y sobre el saldo correspondiente. En los países desarrollados, las proyecciones más negativas con respecto al impacto en el empleo se presentan en Frey y Osborne (2013) quienes estiman que un 47\% de los puestos de trabajo de los Estados Unidos podría sustituirse en un plazo no muy extenso. Cuestionando estos resultados, desde el "enfoque tareas" se argumenta que para desempeñarse en una ocupación específica las personas desarrollan una variedad de tareas, y que si se empiezan a utilizar nuevas tecnologías y se sustituye el trabajo humano en algunas de ellas, en muchos casos esto no necesariamente implica que se elimina el puesto de trabajo (Autor, 2013 y 2015). En vista de que, aparentemente, hay una tendencia a que los trabajadores se desempeñen en un número creciente de tareas, sustituir empleos enteros por tecnologías que automatizarían tareas específicas se transforma en un proceso más complejo ${ }^{7}$. En consecuencia, más bien, surgiría una nueva combinación de maneras en que estas tareas se ejecutan (Akçomak, Kok y Rojas-Romagosa, 2016). Lógicamente, las estimaciones sobre la destrucción de puestos de trabajo que se basan en este enfoque llegan a magnitudes más moderadas. Por ejemplo, Arntz, Gregory y Zierahn (2016) estiman la proporción de puestos de trabajo con alto riesgo de sustitución en los países de la Organización de Cooperación y Desarrollo Económicos (OCDE) en un 9\%.

McKinsey Global Institute (2017a) también toma en cuenta la diferenciación entre ocupaciones y tareas, y analiza a nivel global el potencial de sustitución de 2.000 actividades que se desarrollan en 800 ocupaciones. Encuentra que alrededor la mitad de estas actividades pueden ser automatizadas por tecnologías ya existentes (entre un $41 \%$ y un $56 \%$ de las actividades, según el país). Sin embargo, menos del $5 \%$ de las ocupaciones pueden ser sustituidas enteramente, mientras que un $60 \%$ de ellas abarcan un mínimo del 30\% de actividades automatizables y son, por lo tanto, objeto potencial de transformaciones profundas, más que sustituciones. El escenario base de este informe es que el proceso de automatización de alrededor de un $50 \%$ de las actividades puede darse hasta 2055, pero los autores subrayan que este horizonte depende de una serie de factores de contexto. En un estudio posterior McKinsey Global Institute (2017b), el mismo grupo de autores define como su escenario central que hasta 2030 un 15\% de las horas trabajadas actualmente a nivel global se automatizarían, pero con un rango amplio de entre solo poco más del $0 \%$ y el $30 \%$.

¿Qué se puede decir sobre América Latina? Las proyecciones para la región son aún escasas. El Banco Mundial (2016) aplica la metodología de Frey y Osborne (2013) y halla que en los 11 países latinoamericanos analizados, entre un $62 \%$ de los empleos de la Republica Dominicana y un $75 \%$ de El Salvador y Guatemala son susceptibles a una posible automatización, con un promedio del $67 \%$ en los países latinoamericanos cubiertos (en comparación con un 57\% en los países de la OCDE). Si se

\footnotetext{
7 Becker y Muendler (2014) detectaron un marcado aumento de las tareas que los trabajadores alemanes declaran: 1,67 en 1979 a 7,24 en 2006, en promedio. La creación de cadenas globales de suministro que descomponen el proceso de producción en pasos especializados obraría en contra de esta tendencia (ver OIT, 2016a).
} 
toma en cuenta el rezago en la adaptación de nuevas tecnologías que caracterizan a países menos desarrollados, el rango baja a un todavía elevado nivel de entre un $40 \%$ en Nicaragua y un $65 \%$ en la Argentina, con un promedio del 49\% para los países de la región.

Es interesante observar que el promedio no ajustado de los países latinoamericano es más elevado que el de la OCDE, mientras que si se incorpora un ajuste por los rezagos en los cambios de los procesos productivos a las nuevas tecnologías en países más pobres y de menores niveles de conocimiento, este promedio cae por debajo del nivel de la OCDE.

Basados en la misma metodología de Frey y Osborne (2013), Aboal y Zunino (2017) llegan a resultados similares para la Argentina (64,1\% de probabilidad de automatización) y el Uruguay $(66,4 \%)$, con tasas levemente más elevadas para hombres que para mujeres.

Sobre la base de datos del Foro Económico Mundial y la OIT, para América Latina en su conjunto, en OCDE/CAF/CEPAL (2016, pág. 263) se estima una pérdida neta de empleo de 3,38 millones puestos de trabajo hasta 2030, lo que representa entre el 1 y el $2 \%$ del empleo total. Se proyecta que las pérdidas estarán concentradas en la industria manufacturera con una destrucción de alrededor de 3,5 millones de empleos, las funciones administrativas y de soporte en que se perderían alrededor de 1,3 millones de empleos, y la construcción, con una destrucción de un poco más de un millón de puestos de trabajo. Estas pérdidas no se compensarían con ganancias en el comercio (un poco más de 2 millones de nuevos empleos) y, en menor grado en el transporte (alrededor de medio millón) y otras ramas de actividad.

Siguiendo el enfoque de las tareas, McKinsey Global Institute (2017a) identifica, para un grupo limitado de países latinoamericanos, los porcentajes más altos (más del $51 \%$ de las actividades) de actividades automatizables para Colombia, Costa Rica, México y el Perú, seguidos por el Brasil, así como la Argentina, Chile y la República Dominicana (entre el $45 \%$ y el $47 \%$ ). Como se mencionó, el horizonte de esta proyección es, en el escenario base de los autores, el año 2055. En el escenario central actualizado del McKinsey Global Institute (2017b), entre 2016 y 2030 en los países latinoamericanos analizados se automatizarían alrededor de un $7 \%$ de las actividades (no de los empleos) en el Perú y alrededor del $14 \%$ en el Brasil.

Hay que recordar que el reemplazo de actividades no necesariamente implica la pérdida de un puesto de trabajo. Si se aplica al caso latinoamericano la estimación de McKinsey Global Institute (2017a) con respecto al impacto de las transformaciones a nivel global, según la cual menos de un $5 \%$ de las ocupaciones serían completamente automatizables, en la región se perderían alrededor de 14 millones de empleos en el plazo indicado.

Sin embargo, habría que tomar en cuenta que las transformaciones tecnológicas en cuestión difícilmente afectarían el sector informal de manera directa, dado que en este sector se suelen aplicar tecnologías lejanas de la frontera del conocimiento, por lo que no se vería impactado por la introducción de nuevas tecnologías de punta. De esta manera, si se toma en cuenta esta diferencia estructural en el mercado de trabajo que distingue a América Latina de los países desarrollados y se aplica dicho porcentaje solo a los ocupados en el sector de productividad media o alta, la pérdida sería de aproximadamente 8 millones de empleos. Esto sería el mínimo de los empleos sustituidos, ya que además de estos empleos completamente automatizables, otros, con menos del 100\% de las actividades automatizables, también tienen cierta probabilidad de sustitución

Tomando en cuenta el funcionamiento del mercado de trabajo en América Latina (Weller y Kaldewei, 2014, págs. 68-72), es de suponer que una buena parte de las personas afectadas por esta destrucción de empleos, si no consigue empleo en otras ocupaciones del sector formal, pasaría a emplearse en el sector informal, en lugar de pasar al desempleo o de salir de la fuerza de trabajo. Por lo tanto, el efecto de una masiva pérdida de empleo formal por el cambio tecnológico sería una mayor informalización de la estructura ocupacional. 
Siguiendo estas consideraciones, Weller, Gontero y Campbell (2019) aplican un ajuste a la metodología de Frey y Osborne (2013), para tomar en cuenta las diferencias estructurales entre los mercados laborales latinoamericanos y los de los países desarrollados. Encuentran para un grupo de 12 países de la región que la proporción de los empleos con alto riesgo de sustitución baja de un rango de entre el $25 \%$ en Brasil y el 55\% en El Salvador y Guatemala, que se identifica con la metodología original, a un rango de entre el 5\% en el Estado Plurinacional de Bolivia y el 29\% en el Uruguay con la metodología ajustada, con una mayor proporción de ocupados con alto riesgo de sustitución tecnológica en los países de la región con una mayor PIB per cápita y sectores de productividad alta y media más grandes (véase el gráfico 3). Sin embargo, este menor riesgo reflejaría la persistencia de sectores de baja productividad en los cuales los empleos reflejan más las necesidades de subsistencia de muchos hogares que el grado de capacidad de introducir nuevas tecnologías y la reasignación competitiva de los factores de producción, y los empleos que persistirían en estos sectores serían habitualmente de mala calidad.

\section{Gráfico 3}

América Latina: proporción estimada del empleo con alto riesgo de sustitución tecnológica, método de Frey y Osborne, versiones original y ajustada

(En porcentajes)

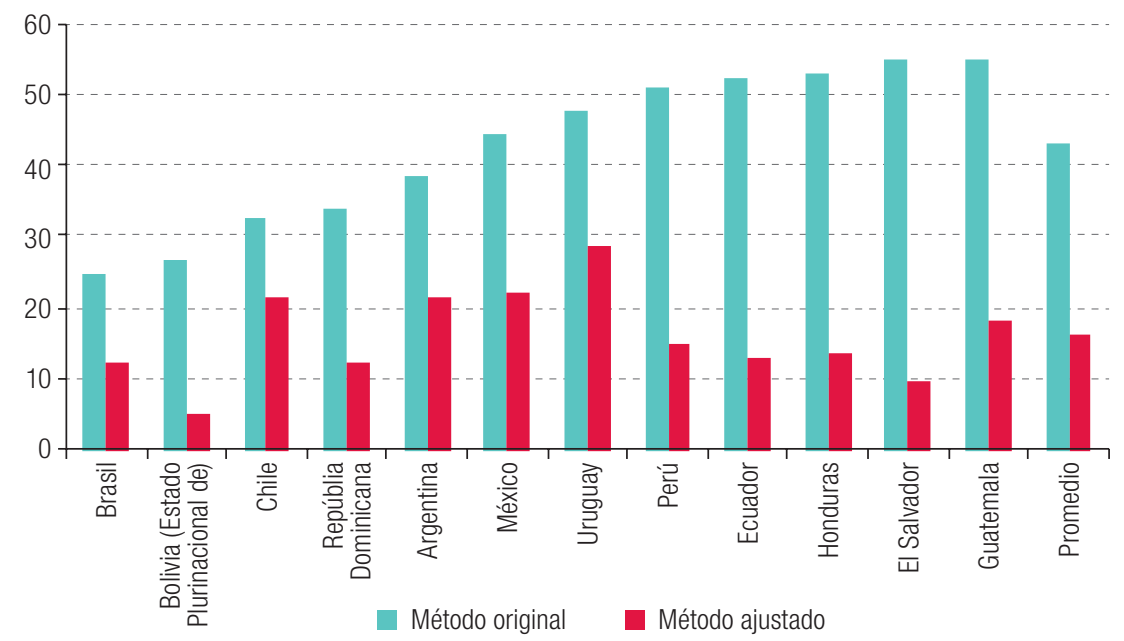

Fuente: Elaboración propia, sobre la base de J. Weller, S. Gontero y S. Campbell, "Cambio tecnológico y empleo: una perspectiva latinoamericana. Riesgos de la sustitución tecnológica del trabajo humano y desafíos de la generación de nuevos puestos de trabajo", serie Macroeconomía del Desarrollo, № 201 (LC/TS.2019/37), Santiago, Comisión Económica para América Latina y el Caribe (CEPAL), 2019.

Los estudios citados, basados generalmente en las consideraciones de expertos o de directivos de empresas, se complementan con las percepciones de los trabajadores en empresas formales. Según la encuesta de Randstad (2016), en los países latinoamericanos analizados (Argentina, Brasil, Chile, México) entre un $45 \%$ de los trabajadores en México y un $60 \%$ en el Brasil considera que ejecutan un trabajo repetitivo o tareas rutinarias que podrían automatizarse. Resulta llamativo que los rangos sobre los empleos potencialmente afectados por la automatización sean muy similares desde la perspectiva de los directivos y de los trabajadores. Dicha proporción es mayor que el promedio global (44\%). Por otra parte, también es más alta la proporción de los trabajadores latinoamericanos que creen que si se automatiza el trabajo repetitivo podrían añadir más valor personal a su trabajo (entre el 70\% y el 74\% en comparación con el $62 \%$ a nivel global). Esta actitud representa obviamente una visión optimista de las transformaciones en que la automatización de tareas rutinarias mejoraría la productividad y calidad del empleo, en vez de destruirlo.

Volviendo a las proyecciones desde la perspectiva de las empresas, es interesante observar que para un plazo muy corto de dos años, muchas empresas latinoamericanas indican que esperan 
un aumento de su planilla como consecuencia de la digitalización. En efecto, los ocho países latinoamericanos incluidos en la encuesta de Manpower Group (2018) informan de saldos positivos respecto a sus planes de contratación correspondientes, y América Latina es, al respecto, la región más optimista de todas. En contraste, por ejemplo, en 7 de un total de 23 países europeos las empresas esperan mayoritariamente una caída del número de ocupados por este motivo, y allí donde el saldo es positivo, en general es menor que en los países latinoamericanos. Esta discrepancia entre las expectativas favorables de corto plazo y las perspectivas más negativas de un plazo más largo puede deberse a que en un período relativamente corto muchas empresas latinoamericanas enfrentan el reto de incorporar nuevas tecnologías a su proceso productivo para mantenerse competitivas, lo que requeriría la contratación de personal especializado en TIC, mientras que el posible impacto ahorrativo de mano de obra se haría sentir posteriormente. En contraste, en los países desarrollados este proceso ya está más avanzado, por lo que esta situación reflejaría el rezago en la introducción y adaptación de nuevas tecnologías que históricamente ha caracterizado el desarrollo latinoamericano.

¿Qué factores influyen, específicamente, en un impacto diferenciado de las nuevas tecnologías en la posible sustitución de empleos en los sectores de productividad alta y media de América Latina? Al respecto, hay que tomar en cuenta que no necesariamente se realizarán todas las sustituciones de tareas que son tecnológicamente factibles (Bensusán, Eichhorst y Rodríguez, 2017). Los factores que influyen en la probabilidad de que un puesto de trabajo sea sustituido se pueden resumir de esta manera:

$$
P S=f\left(+\delta P L_{i},+w_{i},+P T_{i},-C 1_{i},-C 2_{i},+I_{i},+x_{i}\right)
$$

Donde la probabilidad de sustitución (PS) de un puesto de trabajo del sector $i$ aumenta con la diferencia de productividad entre la nueva y la antigua tecnología $\left(\delta P L_{i}\right)$, el costo laboral $\left(w_{i}\right)$ y la proporción de tareas sustituibles en este puesto de trabajo $\left(P T_{i}\right)$. Por otra parte, esta probabilidad disminuye con el costo de introducción de la nueva tecnología $\left(C 1_{i}\right)$ que incluye los costos de adquisición, ajuste, instalación, capacitación, modificación del entorno, entre otros, y con el costo del mantenimiento y actualización $\left(C 2_{i}\right)$.

El impacto de la capacidad de innovación y adaptación tecnológica y organizativa disponible en la economía y el sector específico $\left(I_{i}\right)$ es incierto. Por un lado, una alta capacidad de innovación tiende a conllevar una mayor introducción de tecnologías ahorrativas de mano de obra; por otro lado, si esta capacidad es baja en las ocupaciones que tienen una elevada proporción de actividades automatizables sería difícil lograr combinaciones eficientes entre las nuevas tecnologías y las no automatizables que siguen requiriendo el trabajo humano, por lo que existe el riesgo de una pérdida de competitividad (en bienes y servicios transables) que destruiría los empleos correspondientes.

Finalmente, existe otro conjunto de factores $x_{i}$ cuyo impacto en su conjunto es incierto y que incluye aspectos como requisitos de infraestructura fuera de la empresa, aspectos específicos del sector correspondiente (por ejemplo, expectativas sobre el crecimiento, los cambios en la estructura del mercado y de futuros cambios tecnológicos) y de aspectos específicos de la empresa correspondiente.

En el caso de América Latina, debido a los niveles salariales más bajos, se necesitarían aumentos de la productividad mucho más altos para que la sustitución sea rentable ${ }^{8}$. Además, la capacidad de innovación y adaptación tecnológica y organizativa es, en promedio, menor que en los países desarrollados y, para muchas de las nuevas tecnologías, los costos $C 1_{i}$ y $C 2_{i}$ se ubicarían en niveles más elevados, por ejemplo por los costos relacionados con su importación, así como por los costos de adaptación que pueden ser más elevados en un contexto de mayor escasez de expertos que acompañarían la introducción de las nuevas tecnologías y las actividades posteriores (mantenimiento

8 Cabe recordar que el trabajo en ocupaciones comparables suele generar salarios mucho más bajos en países de menor nivel de desarrollo (Freeman y Oostendorp, 2000). 
y capacitación, entre otras). Finalmente, puede haber requisitos de la infraestructura de entorno que requieren inversiones públicas y que no están garantizados.

Por lo tanto, varios de los factores relevantes para la sustitución reducen la probabilidad que esta suceda en América Latina, de manera que el salto de la sustitución tecnológicamente posible a una sustitución económicamente viable es mayor que en los países desarrollados. Sin embargo, si bien esta consideración lleva a la probabilidad de una sustitución de menos puestos de trabajo en la región, lo que parece una buena noticia, se basa en aspectos relacionados con su atraso económico y en brechas (de infraestructura, capacidad de innovación, productividad o salarios, por ejemplo) que más bien deben cerrarse en el contexto de una estrategia de desarrollo sostenible para la región.

Finalmente, un análisis de la relación entre las transformaciones tecnológicas y el empleo tiene que tomar en cuenta el impacto indirecto. Así, según Berger, Chen y Frey (2017), en los países en desarrollo la generación de empleos en el sector de los servicios a partir de un efecto multiplicador de la generación de empleos cualificados en la industria manufacturera es mayor que en los países desarrollados, lo que podría suavizar en el nivel agregado el impacto del ahorro de mano de obra de las transformaciones tecnológicas.

\section{Aspectos de política para orientar y acompañar las transformaciones tecnológicas}

Si se asume un enfoque contextual condicionado respecto de la introducción de las nuevas tecnologías, surge un papel clave para las políticas públicas que abarca varios campos, desde el desarrollo tecnológico como tal, hasta sus modalidades de introducción en los procesos productivos y de mitigación de sus impactos no deseados pero inevitables.

El impacto real de estas transformaciones tecnológicas en el empleo y sus características depende en gran parte de la manera en que estas se manejan. Por lo tanto, en muchos países (sobre todo desarrollados), estos procesos no se dejan completamente en manos de las fuerzas de mercado sino se han formulado programas específicos para fomentar su desarrollo e introducción al mundo productivo y la sociedad en general (Bensusán, Eichhorst y Rodríguez, 2017), un área de políticas donde América Latina y el Caribe registra grandes rezagos (Cimoli y otros, 2017)9.

En esta sección se discuten dos áreas de política estrechamente relacionadas con el impacto de las transformaciones tecnológicas en el empleo, los retos para regulación laboral y para el desarrollo de las habilidades y competencias requeridas para aprovechar su potencial ${ }^{10}$.

\section{La regulación de las relaciones laborales}

En América Latina se observa una elevada heterogeneidad en las relaciones laborales (Bensusán, Eichhorst y Rodríguez, 2017; Novick, 2018). Esta heterogeneidad es el resultado de diferentes fases históricas. Históricamente, el sector informal ha representado una elevada proporción de las ocupaciones, por lo que, para esta región, debería evitarse la aplicación del concepto del "trabajo atípico", que surgió en contraste con el empleo predominantemente formal en los mercados laborales de los países desarrollados de la posguerra. También dentro del sector formal existe una proporción

9 Para América Latina, la CEPAL (2016) ha propuesto vincular estos aspectos con un incremento de la inversión centrado en un gran impulso ambiental.

10 Otros temas relevantes al respecto son, por ejemplo, la política fiscal (impuesto a los robots, Shiller, 2017), la protección de los afectados por los cambios tecnológicos (ingreso básico universal, Naciones Unidas, 2017) y la reducción de la jornada laboral (Mokyr, Vickers y Ziebarth, 2015). 
relevante de empleos informales, en muchos casos como expresión de estrategias empresariales de reducción de costos. Adicionalmente, durante los años noventa, muchos países latinoamericanos introdujeron nuevas modalidades de trabajo formal, que - más allá del empleo a tiempo completo y a plazo indefinido - ampliaron las opciones formales de contratación a tiempo parcial o a plazo definido.

Finalmente, a partir de las transformaciones tecnológicas en curso, también en América Latina están surgiendo nuevas modalidades de relación laboral, por ejemplo la contratación a través de plataformas en Internet y el trabajo en la "economía colaborativa" (Artecona y Chau, 2017; IE/BID/ FOMIN, 2016). De esta manera, de las transformaciones tecnológicas en curso surge una tendencia a una creciente heterogeneidad de las relaciones laborales, con lo cual se generan nuevos retos para las instituciones laborales.

Algunas de estas nuevas modalidades de trabajo tienden a bajar los ingresos laborales, como el crowdworking en el cual la empresa que busca insumos puede recurrir a una oferta global desregulada. Una modalidad especialmente precaria consiste en que la empresa contratante solo remunera el trabajo que le es más útil, de manera que el trabajo de los otros oferentes queda sin recompensa (winner takes all) (Krull, 2016, pág. 16).

Además de que en muchos casos (obviamente no en todos) los ingresos que se perciben en las nuevas modalidades de trabajo son inestables, se ha destacado que a partir de las nuevas tecnologías han emergido las nuevas formas de control del trabajador (Krull, 2016, págs. 19 y 24). Por una parte, las nuevas tecnologías permiten la generación de una multiplicidad de datos que facilitan un control cada vez más detallado de la actividad de los trabajadores. Por otra parte, un elemento cada vez más relevante al respecto es - por ejemplo, en el caso de los conductores de Uber - la reputación que permite u obstaculiza - en caso de su deterioro-el acceso a oportunidades de trabajo.

Al mismo tiempo, la presión de estar conectado permanentemente a las redes de información tiende a generar niveles de sobrecarga y estrés. Además, la ausencia de un trabajo a tiempo completo puede generar la obligación de una inserción laboral múltiple, dificulta el funcionamiento de las instituciones laborales tradicionales y erosiona los límites entre el mundo del trabajo y de la vida privada (OCDE/ CAF/CEPAL, 2016, pág. 264). En consecuencia, en varios países (incluidos los de América Latina) se han propuesto iniciativas legales para promover la desconexión fuera del horario de trabajo a fin de apoyar equilibrios adecuados entre el trabajo y el descanso y la vida privada (Diario Financiero, 2017a).

La creciente heterogeneidad en las relaciones laborales ha generado un debate sobre si se requiere diseñar regulaciones específicas para las diferentes modalidades de relación laboral existentes entre el trabajador, el cliente, y el empleador o intermediario (empleador tradicional, empleador subcontratista, empresa de plataforma web, entre otros), o si la tarea del regulador debe consistir en determinar a partir de indicadores claves si el trabajador es asalariado (aunque con ciertas especificidades) o independiente (trabajador por cuenta propia) (Bensusán, Eichhorst y Rodríguez, 2017; OIT, 2016b). Ambas opciones conllevan tareas complejas. En el primer caso, el desafío consiste en diseñar regulaciones para las nuevas modalidades que respondan al mandato de las instituciones laborales, combinando adecuadamente eficiencia y protección. En el segundo caso habría que asegurar que el trabajador reciba los beneficios que la legislación laboral considera para los asalariados y avanzar en la formalización del trabajo independiente, estableciendo claramente los deberes y derechos relacionados con esta categoría ocupacional.

Por otra parte, también para los asalariados y las instituciones laborales correspondientes surgen nuevos retos. Por ejemplo, cabe preguntarse si la automatización de grandes partes del proceso productivo debilita el derecho de huelga (Diario Financiero, 2017b).

El análisis sobre el impacto de las transformaciones tecnológicas en las relaciones laborales y la elaboración de la regulación correspondiente debe ser un proceso participativo en el cual deben involucrarse tanto sindicatos y trabajadores activos en actividades caracterizadas por nuevos tipos de 
relaciones laborales, como las propias empresas. Con frecuencia se sostiene que la regulación de estas actividades puede obstaculizar el aprovechamiento del potencial para el aumento de la productividad. Sin embargo, aprovechar este potencial con una reducción de la calidad del empleo dificultaría el establecimiento de círculos virtuosos entre la productividad y los ingresos laborales y aspectos no salariales de la calidad del empleo, que sirve de base para la sostenibilidad económica y social del modelo económico imperante.

En vista de que no hay soluciones óptimas únicas para la implementación de las nuevas tecnologías (como se argumentaría desde un enfoque de determinismo tecnológico) esta debe ser el resultado de ejercicios participativos que conforman un marco para procesos de aprendizaje. Por ejemplo, Bensusán, Eichhorst y Rodríguez (2017) subrayan que la aplicación de las regulaciones laborales a las nuevas modalidades de trabajo debe basarse en la negociación y el diálogo ${ }^{11}$. En este contexto, Sundararajan (2017) promueve la reformulación del contrato social que debería tomar en cuenta la creciente heterogeneidad de la fuerza laboral, y Adigital/GOVUP (2017) plantean, entre otras cosas, la importancia de la colaboración entre organismos públicos y plataformas para facilitar la recaudación de los tributos y de las cotizaciones a la seguridad social; la posibilidad de que las plataformas funcionen con modelos mixtos de relaciones laborales, en acuerdo con los trabajadores; la clara delimitación del trabajo por cuenta propia; el establecimiento de pisos mínimos respecto a ingresos y protección social; los seguros de responsabilidad por daños a terceros, y las mejoras en el acceso a la información sobre derechos y obligaciones.

Un proceso participativo tiende a generar resultados alejados de los extremos (desregulación total o regulación extremadamente rígida), que suelen no ser socialmente aceptables. Por ejemplo, por una parte la falta de regulación en el ámbito del transporte de personas organizadas por empresas de plataforma web ha generado movimientos de los propios trabajadores que protestan por las condiciones laborales no aceptables y reclaman beneficios adecuados; por otra parte, la evidencia indica que muchos de los trabajadores activos en las nuevas modalidades valoran la flexibilidad inherente a ellas ${ }^{12}$. También hay que tomar en cuenta que la valoración del pago percibido por las nuevas modalidades difiere si representa un ingreso complementario a un ingreso base, derivado de actividades en algunas horas de disponibilidad, o si es el ingreso principal, en cuyo caso aspectos como su variabilidad tienen una gravitación mucho mayor. Por último, se observa que entre los trabajadores se ha detectado una creciente heterogeneidad respecto a los valores que orientan sus preferencias en relación con el trabajo (BMAS, 2017, págs. 36-37).

\section{Habilidades y competencias para aprovechar el potencial de las nuevas tecnologías}

Si bien sigue habiendo una gran incertidumbre respecto al impacto de las transformaciones tecnológicas en el empleo, existe un amplio consenso en el sentido de que la educación, la formación profesional y la capacitación son instrumentos clave para el aprovechamiento del potencial de esas transformaciones y para limitar su impacto negativo ${ }^{13}$. Respecto al aprovechamiento de este potencial, se ha argumentado que el débil incremento de la productividad laboral que se registra en la actualidad en los países desarrollados a pesar de la introducción de las nuevas tecnologías se debe a que tanto a nivel gerencial

\footnotetext{
${ }^{11}$ En la misma línea, el Ministerio de Trabajo de Alemania destaca la importancia de la cooperación de los actores sociales y concluye que lo deseable es una fase de aprendizaje conjunto "dentro de la transformación y a partir de ella", así como probar nuevos conceptos de manera experimental (BMAS, 2017, pág. 13).

12 Según una encuesta del sindicato alemán del sector servicios ver.di entre miembros que trabajan por cuenta propia, estos expresan un elevado nivel de satisfacción en el trabajo, pero están preocupados por aspectos como la inestabilidad de los ingresos y la jubilación (Koch, 2017).

${ }^{13}$ Véase, por ejemplo, Fiszbein, Cosentino y Cumsille (2016), OCDE (2017), Foro Económico Mundial (2018) y Manpower Group (2018).
} 
como a nivel de los trabajadores de producción todavía escasean los conocimientos y competencias para aprovecharlo (Baily y Montalbano, 2016) ${ }^{14}$. Si fuera cierto, esto supondría que una vez superados los cuellos de botella a través de las diferentes formas de aprendizaje se podrían alcanzar importantes ganancias de productividad, con lo cual el impacto en la productividad tendría rezagos respecto al momento del inicio de la introducción de las innovaciones que serían similares a los observados en otras revoluciones tecnológicas.

Por otra parte, a raíz de la destrucción de empleos y la desaparición de ciertas ocupaciones una cantidad no determinada de personas perderían buena parte de su capital humano, lo que haría necesario su recapacitación para otras ocupaciones. La experiencia ha demostrado que no se trata de una tarea fácil (Miller, 2017). Un enfoque interesante se presenta al respecto en Foro Económico Mundial (2018), donde se sugiere recapacitar a las personas que pierden su empleo para su reinserción en ocupaciones que, según ciertos criterios, son parecidas a las que desempeñaban previamente.

En general, hay consenso que para una inserción exitosa en puestos de trabajo caracterizados por la utilización de las nuevas tecnologías se requiere principalmente habilidades no rutinarias, sobre todo cognitivas. Sin embargo, estudios recientes han destacado que aún más importante para una exitosa inserción en estos empleos es la combinación de estas habilidades con competencias interpersonales y sociales como la capacidad de resolver problemas, la comunicación y el trabajo en equipo, que no pueden ser sustituidas fácilmente por las nuevas tecnologías (OCDE, 2017; Edin y otros, 2017; Manpower Group, 2018).

Lamentablemente, en los estudios comparativos internacionales los países de América Latina y el Caribe presentan grandes rezagos en comparación tanto con países desarrollados como con algunos países emergentes en lo referente a las habilidades básicas (que mide la prueba PISA) y a las competencias relacionadas con las demandas del mundo laboral (que mide la prueba PIAAC) (Fiszbein, Cosentino y Cumsille, 2016; OCDE, 2017).

Además, tanto en países desarrollados con en América Latina y el Caribe se ha destacado el problema del desajuste de cualificaciones (mismatch) entre las cualificaciones que se adquieren en los sistemas de educación y formación y la demanda requerida en los procesos productivos, lo que se expresa, por ejemplo, en las quejas recurrentes de las empresas de que no encuentran personal con las competencias requeridas ${ }^{15}$. Por otro lado, América Latina y el Caribe muestra altos niveles de desempleo juvenil, incluso en los estratos altos de educación. Para abordar este problema es preciso identificar mejor la demanda actual y futura de cualificaciones (Novick, 2017), realizar el ajuste correspondiente de los sistemas de educación y formación profesional y capacitación, y lograr que todos los interesados tengan acceso a esa información, especialmente los jóvenes que están tomando decisiones sobre sus futuros estudios (Gontero y Zambrano, 2018). Sin embargo, tal como sostienen Rathelot y Van Rens (2017), sería también importante plantearse mecanismos para mejorar el funcionamiento de los mercados laborales, los cuales deberían reflejar de manera más nítida - a través de salarios más altos - la escasez en las ocupaciones correspondientes.

Por otra parte, el atraso en la adquisición de algunas de las competencias requeridas para el manejo eficiente de las nuevas tecnologías también pone de manifiesto una brecha generacional, dado que a este respecto las generaciones más jóvenes son muchos más competentes que las anteriores (OCDE, 2016). Para fortalecer las competencias de las personas ya insertas al mercado de trabajo habría que asumir un enfoque centrado en las empresas y a nivel interempresas (OCDE/OIT, 2017). En efecto, capacitar a las personas ocupadas en las mismas empresas facilita el ajuste de sus competencias a la

\footnotetext{
14 Incluso para los países miembros de la OCDE se han identificado grandes brechas entre la futura demanda de competencias y las cualificaciones de la fuerza laboral actual. Específicamente, un 56\% de los adultos de la OCDE tienen solo habilidades básicas respecto a las TIC, o no tienen ninguna (OCDE, 2016).

${ }^{15}$ Véanse, por ejemplo, los resultados de la encuesta de talentos de Manpower [en línea] https://www.manpowergroup.com/ talent-shortage-2016.
} 
demanda existente. A la vez, a raíz de la puesta en práctica de iniciativas sectoriales coordinadas por ejemplo por cámaras u otras asociaciones de empresas, se puede enfrentar las preocupaciones de las empresas individuales de que podrían perder a sus trabajadores una vez que estos hayan adquirido las nuevas competencias. Un enfoque tripartito que incluya a las organizaciones de los trabajadores en la identificación de la demanda y los mecanismos de capacitación ha mostrado - por ejemplo en los sistemas de certificación de competencias - que puede contribuir a mejorar la efectividad de estos procesos. Las opciones de aprendizaje en línea repercutirían positivamente en los costos y en la efectividad de esa capacitación (Frey, 2017).

La subrepresentación de las mujeres en ocupaciones con perspectiva de expansión en el contexto de la introducción de las nuevas tecnologías, así como la amenaza correspondiente de una ampliación de las brechas ya existentes entre hombres y mujeres en el mercado laboral, ponen de manifiesto el reto de asumir una perspectiva de género en los esfuerzos para fortalecer la formación y capacitación. Al respecto, se observan dos aspectos positivos. Primero, mientras que entre las personas de 55 a 65 años la capacidad de solucionar problemas en un entorno intensivo en tecnología es, en general, mayor para los hombres, en el grupo de 25 a 34 años los resultados son mixtos, con un número similar de países con ventajas para mujeres que para hombres, lo que indica que en las generaciones más jóvenes la cercanía a las nuevas tecnologías y por lo tanto la capacidad de aprendizaje en este entorno, no es diferente entre hombres y mujeres (OCDE, 2017, pág. 107). Segundo, la prima salarial para realizar tareas intensivas en tecnología de información y comunicación es mayor para las mujeres que para los hombres, lo que podría incentivar a más mujeres a adquirir las competencias correspondientes (OCDE, 2017, pág. 48) ${ }^{16}$.

\section{Comentarios finales}

En este artículo se sostiene que para la evaluación del posible impacto de las transformaciones tecnológicas en el empleo debería aplicarse el enfoque contextual condicionado, dado que los enfoques compensatorio, determinista y contextual simple no lograrían captar la complejidad de los procesos tecnológicos, económicos y sociales relacionados con estas transformaciones ni harían un aporte adecuado al análisis de la políticas públicas que fomenten el aprovechamiento de su potencial para el desarrollo y limiten su potencial impacto negativo.

Dicho esto, tanto a escala mundial como en América Latina persiste un elevado grado de incertidumbre sobre el impacto de los nuevos avances tecnológicos en el empleo y sus características. Por una parte, habrá destrucción de empleos, que se sustituirán por nuevas tecnologías o que se perderán por una reestructuración de las cadenas globales de valor. Según estimaciones que parecen realistas, la destrucción neta ascendería a entre el 1\% y el 2\% del empleo total (3,38 millones de empleos) para 2030 y al 5\% del empleo formal (alrededor de 8 millones de empleos) para 2055. Esto, sin embargo, sería el piso mínimo de la destrucción de empleos, establecido respecto a los puestos de trabajo que contienen una alta proporción de ocupaciones automatizables. Desde el enfoque contextual condicionado se plantea que más allá de los empleos con un elevado componente de tareas automatizables el resultado en términos de destrucción y generación de empleo depende de las acciones que adopten los actores, si bien no lo pueden hacer con absoluta libertad sino condicionados por las características de las tecnologías que se hacen disponibles a lo largo del tiempo. De esta manera, se perdería una cantidad adicional de empleos si no se logra adaptar los procesos productivos $-\mathrm{y}$, especialmente, el trabajo humano - al nuevo contexto tecnológico y, al revés, si no se adaptan las nuevas tecnologías al ser humano.

\footnotetext{
${ }^{16}$ Esto no significa que estas mujeres perciban salarios más altos que sus pares masculinos, sino que el aumento salarial, en comparación con otras mujeres, es mayor que en el caso de los hombres.
} 
Por otro lado, en América Latina y el Caribe la introducción de las nuevas tecnologías enfrenta obstáculos tales como los menores costos laborales. Estos últimos hacen necesario que las nuevas tecnologías generen un mayor salto de productividad para que su introducción sea rentable. También constituyen trabas los costos de introducción, mantenimiento y actualización de esas tecnologías, las limitadas capacidades de innovación y adaptación, y la existencia de una infraestructura deficiente. Esos obstáculos hacen que las transiciones sean más lentas, lo que por un lado atenúa el impacto en la destrucción directa de empleo, pero por otro frena el aprovechamiento del potencial de esas tecnologías y, de esta manera, la generación de nuevos puestos de trabajo.

Al parecer, más que en la destrucción completa de empleos, el efecto más amplio de las transformaciones tecnológicas consistirá en la transformación de la manera de trabajar y de los empleos. Ello, a partir de la incorporación de nuevas tecnologías en la ejecución de ciertas tareas, las cuales habría que integrar de una manera diferente en el conjunto de las actividades que se ejecutan en los puestos de trabajo. Con ese propósito, los sistemas de educación y formación profesional y capacitación han de ajustarse a los profundos cambios que llevan aparejadas las transformaciones tecnológicas para la demanda de cualificaciones. Por otro lado, se deben afrontar las brechas de conocimientos y competencias que, según los estudios comparativos, colocan a América Latina y el Caribe en situación de desventaja (OIT, 2016c). En este contexto y en vista del impacto diferenciado que la introducción de las nuevas tecnologías tendría para hombres y mujeres y de los sesgos existentes en la educación y formación, es indispensable adoptar un enfoque de género.

Por otra parte, las nuevas tecnologías amplían el espacio para una mayor variedad de relaciones laborales, lo que genera nuevos desafíos para el diseño de las instituciones laborales, con tal de que se aproveche el potencial productivo de estas nuevas formas de trabajar sin sacrificar derechos laborales y calidad de empleo. Específicamente en América Latina, una pérdida de empleos de mediana y alta productividad del sector formal daría lugar a un aumento del empleo informal en sectores de baja productividad, más que a una caída absoluta del número de ocupados.

Además, hay que tomar en cuenta que las transformaciones tecnológicas se desenvuelven en un contexto en que ocurren también otras transformaciones. En concreto, los cambios demográficos y su impacto en el envejecimiento de muchos de los países de la región generan nuevas necesidades que requieren respuestas integradas; por otra parte, es necesario que se produzcan importantes transformaciones de la matriz energética y de las pautas de producción y consumo para asegurar la sostenibilidad del crecimiento (CEPAL, 2016).

En efecto, aún sin las amenazas que provendrían de las nuevas tecnologías, América Latina habría enfrentado el desafío de transformar su estructura productiva a través de un cambio estructural progresivo. Enfocar las políticas públicas hacia esta meta supone establecer un marco para la incorporación productiva de estas tecnologías. En este contexto las nuevas tecnologías podrían permitir un salto en el proceso de desarrollo, ya que sería posible acercarse a la frontera de producción sin previamente tener que volverse competitivo en áreas productivas dominadas por tecnologías tradicionales.

La complejidad de este desafío y la sostenibilidad de las políticas requieren un enfoque participativo, acorde con los pactos para la igualdad, la sostenibilidad y el cambio estructural propuestos por la CEPAL (2014).

Para concluir, a nivel agregado es difícil estimar el impacto neto de las transformaciones tecnológicas, tanto por la incertidumbre respecto a la cantidad de puestos de trabajo que se eliminarán, como respecto al número de nuevos empleos que se generarán. Sin embargo, queda bien establecido el reto de potenciar la interacción entre los seres humanos y las tecnologías y de adaptar las habilidades y competencias de la fuerza laboral a las nuevas exigencias y oportunidades, así como de hacer frente a la amenaza de la profundización de las desigualdades existentes y emergentes, lo que implica nuevos desafíos para las instituciones laborales. 


\section{Bibliografía}

Aaronson, D. y B. J. Phelan (2017), "Wage shocks and the technological substitution of low-wage jobs", Working Paper, No 2017-3, Chicago, Banco de la Reserva Federal de Chicago.

Aboal, D. y G. Zunino (2017), “Innovación y habilidades en América Latina”, Integración y Comercio, № 42, Washington, D.C., Banco Interamericano de Desarrollo (BID).

Acemoglu, D. y D. Autor (2012), "What does human capital do? A review of Goldin and Katz's The Race between Education and Technology", Journal of Economic Literature, vol. 50, N², Nashville, Asociación Estadounidense de Economía.

Acemoglu, D. y P. Restrepo (2017), "Robots and jobs: evidence from US labor markets", NBER Working Paper, N²3285, Cambridge, Oficina Nacional de Investigaciones Económicas (NBER). (2016), "The race between machine and man: implications of technology for growth, factor shares and employment", NBER Working Paper, N²2252, Cambridge, Oficina Nacional de Investigaciones Económicas (NBER).

Adigital (Asociación Española de la Economía Digital)/GOVUP (2017), Trabajo en plataformas digitales. Análisis y propuestas de regulación [en línea] https://www.adigital.org/informes-estudios/trabajo-enplataformas-digitales/.

Akçomak, S., S. Kok y H. Rojas-Romagosa (2016), "Tecnología, deslocalización y tareas definitorias de las ocupaciones en el Reino Unido", Revista Internacional del Trabajo, vol. 155, № 2, Hoboken, Wiley.

Arntz, M., T. Gregory y U. Zierahn (2016), "The risk of automation for jobs in OECD countries. A comparative analysis", OECD Social, Employment and Migration Working Paper, N 189, París, OECD Publishing.

Artecona, R. y T. Chau (2017), "Labour issues in the digital economy", Studies and Perspectives seriesOficina de la CEPAL en Washington, № 17 (LC/TS.2017/66), D.C., Comisión Económica para América Latina y el Caribe (CEPAL).

Autor, D. (2015), "Why are there still so many jobs? The history and future of workplace automation", The Journal of Economic Perspectives, vol. 29, N³, Nashville, Asociación Estadounidense de Economía. (2013), "The 'task approach' to labor markets: an overview", NBER Working Paper, № 18711, Cambridge, Oficina Nacional de Investigaciones Económicas (NBER).

Baily, M. N. y N. Montalbano (2016), "Why is US productivity growth so slow? Possible explanations and policy responses", Hutchins Center Working Paper, № 22, Washington, D.C., Hutchins Center on Fiscal \& Monetary Policy at Brookings.

Banco Mundial (2016), World Development Report 2016: Digital Dividends, Washington, D.C.

Becker, S. O. y M. A. Muendler (2014), "Trade and tasks: an exploration over three decades in Germany" [en línea] http://econweb.ucsd.edu/muendler/papers/tradtask.pdf.

Bensusán, G., W. Eichhorst y J. M. Rodríguez (2017), "Las transformaciones tecnológicas y sus desafíos para el empleo, las relaciones laborales y la identificación de la demanda de cualificaciones", Documentos de Proyectos (LC/TS.2017/111), Santiago, Comisión Económica para América Latina y el Caribe (CEPAL).

Berger, T., C. Chen y C. B. Frey (2017), "Cities, industrialization, and job creation: evidence from emerging economies" [en línea] https://www.oxfordmartin.ox.ac.uk/downloads/JEG_Manuscript.pdf.

Bessen, J. (2015), "Toil and technology", Finance \& Development, vol. 52, N 1, Washington, D.C., Fondo Monetario Internacional (FMI).

BMAS (Ministerio Federal de Trabajo y Asuntos Sociales) (2017), Weißbuch Arbeiten 4.0, Berlín [en línea] https://www.bmas.de/SharedDocs/Downloads/DE/PDF-Publikationen/a883-weissbuch.pdf? blob=publicationFile\&v=9.

CEPAL (Comisión Económica para América Latina y el Caribe) (2017), Resumen de la presidencia del Foro de los Países de América Latina y el Caribe sobre el desarrollo sostenible [en línea] https://foroalc2030.cepal. org/2017/es/documentos/resumen-la-presidencia-foro-paises-america-latina-caribe-desarrollo-sostenible. (2016), Horizontes 2030. La igualdad en el centro del desarrollo sostenible (LC/G.2660/ Rev.1), Santiago. (2014), Pactos para la igualdad: hacia un futuro sostenible (LC/G.2586(SES.35/3)), Santiago.

Cimoli, M. y otros (eds.) (2017), Políticas industriales y tecnológicas en América Latina (LC/TS.2017/91), Santiago, Comisión Económica para América Latina y el Caribe (CEPAL)/Agencia Alemana de Cooperación Internacional (GIZ).

Diario Financiero (2017a), “Diputados promueven cambio para establecer 'desconexión digital' después del trabajo", Santiago, 3 de febrero. 
(2017b), “¿Nuevos tiempos? Los robots ponen en jaque el derecho a huelga de los trabajadores”, Santiago, 17 de mayo.

Edin, P.-A. y otros (2017), "The rising return to non-cognitive skill”, IZA Discussion Paper, № 10914, Bonn, Instituto de Economía Laboral (IZA).

Fiszbein, A., C. Cosentino y B. Cumsille (2016), The Skills Development Challenge in Latin America: Diagnosing the Problems and Identifying Public Policy Solutions, Washington, D.C., Inter-American Dialogue and Mathematica Policy Research.

FMI (Fondo Monetario Internacional) (2017), World Economic Outlook 2017: Gaining Momentum?, Washington, D.C.

Foro Económico Mundial (2018), Towards a Reskilling Revolution: A Future of Jobs for All, Colonia/Ginebra. (2016), The Future of Jobs: Employment, Skills and Workforce Strategy for the Forth Industrial Revolution, Colonia/Ginebra.

Freeman, R. B. y R. H. Oostendorp (2000), "Wages around the world: pay across occupations and countries", NBER Working Paper, № 8058, Cambridge, Oficina Nacional de Investigaciones Económicas (NBER).

Frey, C. B. (2017), "¿Trabajadores versus robots? Cómo ganar el duelo más importante del siglo XXI", Integración y Comercio, № 42, Washington, D.C., Banco Interamericano de Desarrollo (BID).

Frey, C. B. y M. A. Osborne (2013), "The future of employment: how susceptible are jobs to computerisation?", Technological Forecasting and Social Change, vol. 114, Amsterdam, Elsevier.

Gontero, S. y M. J. Zambrano (2018), "La construcción de sistemas de información sobre el mercado laboral en América Latina", serie Macroeconomía del Desarrollo, № 193 (LC/TS.2018/18), Santiago, Comisión Económica para América Latina y el Caribe (CEPAL).

Gordon, R. (2016), The Rise and Fall of American Growth: The U.S. Standard of Living since the Civil War, Princeton, Princeton University Press.

Graetz, G. y G. Michaels (2017), "Is modern technology responsible for jobless recoveries?", American Economic Review, vol. 107, № 5, Nashville, Asociación Estadounidense de Economía.

Hathaway, I. y M. Muro (2016), "Tracking the gig economy: new Numbers", Brookings, 13 de octubre [en línea] https://www.brookings.edu/research/tracking-the-gig-economy-new-numbers.

IE/BID/FOMIN (Instituto de Empresa/Banco Interamericano de Desarrollo/Fondo Multilateral de Inversiones) (2016), Economía colaborativa en América Latina, Madrid.

Kinkel, S. (2012), "Trends in production relocation and backshoring activities: changing patterns in the course of the global economic crisis", International Journal of Operations \& Production Management, vol. 32, $N^{\circ}$ 6, Bingley, Emerald Group Publishing.

KPMG International Cooperative (2016), Rise of the Humans. The integration of digital and human labor, Amstelveen [en línea] https://assets.kpmg.com/content/dam/kpmg/xx/pdf/2016/11/rise-of-the-humans.pdf.

Koch, H. (2017), "Zufrieden, aber kaum abgesichert", Taz (Die Tageszeitung), Berlín, 20 de abril [en línea] http://taz.de/Verdi-Umfrage-zur-Selbststaendigkeit/!5402549/.

Krull, S. (2016), "El cambio tecnológico y el nuevo contexto del empleo: tendencias generales y en América Latina", Documentos de Proyectos (LC/W.725), Santiago, Comisión Económica para América Latina y el Caribe (CEPAL).

Manpower Group (2018), Skills Revolution 2.0. Robots Need not Apply: Human Solutions for the Skills Revolution [en línea] https://www.manpowergroup.com/wps/wcm/connect/59db87a7-16c6-490d-ae701bd7a322c240/Robots_Need_Not_Apply.pdf?MOD=AJPERES.

(2017), The Skills Revolution: Digitalization and Why Skills and Talent Matter [en línea] https://www. manpowergroup.com/wcm/connect/5943478f-69d4-4512-83d8-36bfa6308f1b/MG_Skills_Revolution_lores. pdf?MOD=AJPERES\&amp;CACHEID=ROOTWORKSPACE-5943478f-69d4-4512-83d8-36bfa6308f1bmy5RaOM.

McKinsey Global Institute (2017a), A Future that Works: Automation, Employment, and Productivity, McKinsey \& Company.

(2017b), Jobs Lost, Jobs Gained: Workforce Transitions In A Time of Automation, McKinsey \& Company.

Miller, C. C. (2017), "Why Men Don't Want the Jobs Done Mostly by Women", The New York Times, Nueva York, 4 de enero [en línea] https://www.nytimes.com/2017/01/04/upshot/why-men-dont-want-the-jobsdone-mostly-by-women.html?_r=0.

Mokyr, J., C. Vickers y N. L. Ziebarth (2015), "The history of technological anxiety and the future of economic growth: Is this time different?", The Journal of Economic Perspectives, vol. 29, № 3, Nashville, Asociación Estadounidense de Economía. 
Naciones Unidas (2017), Informe del Relator Especial sobre la extrema pobreza y los derechos humanos (A/HRC/35/26), Consejo de Derechos Humanos, 22 de marzo.

Novick, M. (2018), "El mundo del trabajo: cambios y desafíos en materia de inclusión", serie Políticas Sociales, № 228 (LC/TS.2018/2), Santiago, Comisión Económica para América Latina y el Caribe (CEPAL).

_ (2017), "Metodologías aplicadas en América Latina para anticipar demandas de las empresas en materia de competencias técnicas y profesionales", serie Macroeconomía del Desarrollo, № 187 (LC/TS.2017/37), Santiago, Comisión Económica para América Latina y el Caribe (CEPAL).

OCDE (Organización de Cooperación y Desarrollo Económicos) (2017), OECD Science, Technology and Industry Scoreboard 2017: The Digital Transformation, París, OECD Publishing. (2016), Skills for a Digital World: Policy Brief on the Future of Work, París, OECD Publishing.

OCDE/CAF/CEPAL (Organización de Cooperación y Desarrollo Económicos/Banco de Desarrollo de América Latina/Comisión Económica para América Latina y el Caribe) (2016), Perspectivas económicas de América Latina 2017: juventud, competencias y emprendimiento, París, OECD Publishing.

OCDE/OIT (Organización de Cooperación y Desarrollo Económicos/Organización Internacional del Trabajo) (2017), Better Use of Skills in the Workplace: Why It Matters for Productivity and Local Jobs, París, OECD Publishing.

OIT (Organización Internacional del Trabajo) (2016a), "Los cambios tecnológicos y el trabajo en el futuro: cómo lograr que la tecnología beneficie a todos", Nota Informativa, № 1, Ginebra.

- (2016b), "Un futuro lleno de retos para las relaciones de trabajo: ¿es momento de consolidar o de buscar alternativas?", Nota Informativa, № 3, Ginebra.

_ (2016c), Panorama Laboral 2016: América Latina y el Caribe, Lima. (2015), World Employment and Social Outlook: Trends 2015, Ginebra.

Pratt, G. A. (2015), "Is a Cambrian explosion coming for robotics?", The Journal of Economic Perspectives, vol. 29, №3, Nashville, Asociación Estadounidense de Economía.

Randstad (2016), Randstad Workmonitor 2016 - Q4 [en línea] https://cdn2.hubspot.net/hubfs/481927/ Randstad\%20Workmonitor_global_report_Dec2016\%20(8).pdf?submissionGuid=959a4dce-65d7-485ab38c-829ce90182d2.

Rathelot, R. y T. van Rens (2017), "Rethinking the skills gap", IZA World of Labor, № 391, Bonn, Instituto de Economía Laboral (IZA).

Saxer, M. (2017), “¿Cómo crear empleos en la era digital?”, Nueva Sociedad, Buenos Aires, abril [en línea] http://nuso.org/articulo/como-crear-empleos-en-la-era-digital/.

Schwab, K. (2016), "The Fourth Industrial Revolution: what it means, how to respond", World Economic Forum, Ginebra, 14 de junio [en línea] https://www.weforum.org/agenda/2016/01/the-fourth-industrialrevolution-what-it-means-and-how-to-respond/.

Shiller, R. (2017), "Robotization without taxation?", Project Syndicate, Praga, 22 de marzo [en línea] https://www. project-syndicate.org/commentary/temporary-robot-tax-finances-adjustment-by-robert-j--shiller-2017-03.

Sundararajan, A. (2017), "Capitalismo colaborativo", Integración y Comercio, № 42, Washington, D.C., Banco Interamericano de Desarrollo (BID).

Tarabusi, C. C. (1997), "Technology and employment: the role of organisational change and learning", Science Technology Industry Review, № 20, París, OECD Publishing.

Weller, J., S. Gontero y S. Campbell (2019), "Cambio tecnológico y empleo: una perspectiva latinoamericana. Riesgos de la sustitución tecnológica del trabajo humano y desafíos de la generación de nuevos puestos de trabajo", serie Macroeconomía del desarrollo, № 201 (LC/TS.2019/37), Santiago, Comisión Económica para América Latina y el Caribe (CEPAL).

Weller, J. y C. Kaldewei (2014), "Crecimiento económico, empleo, productividad e igualdad", Inestabilidad y desigualdad. La vulnerabilidad del crecimiento en América Latina y el Caribe, J. A. Fuentes (ed.), Libros de la CEPAL, № 128 (LC/G.2618-P), Santiago, Comisión Económica para América Latina y el Caribe (CEPAL).

Wolf, M. (2016), "The tide of globalisation is turning", Londres, Financial Times, 6 de septiembre [en línea] https://www.ft.com/content/87bb0eda-7364-11e6-bf48-b372cdb1043a. 